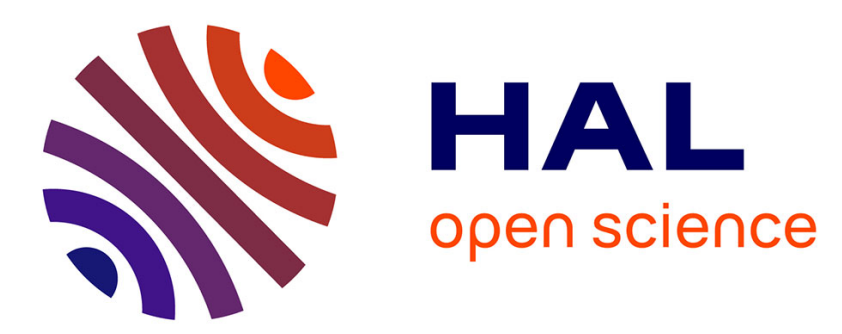

\title{
A semantic-based decision support platform to assist products' eco-labeling process
}

Da Xu, Mohamed Hedi Karray, Bernard Archimède

\section{To cite this version:}

Da Xu, Mohamed Hedi Karray, Bernard Archimède. A semantic-based decision support platform to assist products' eco-labeling process. Industrial Management \& Data Systems, 2017, 117 (7), pp.13401361. 10.1108/IMDS-09-2016-0405 . hal-02359711

\section{HAL Id: hal-02359711 \\ https://hal.science/hal-02359711}

Submitted on 12 Nov 2019

HAL is a multi-disciplinary open access archive for the deposit and dissemination of scientific research documents, whether they are published or not. The documents may come from teaching and research institutions in France or abroad, or from public or private research centers.
L'archive ouverte pluridisciplinaire $\mathbf{H A L}$, est destinée au dépôt et à la diffusion de documents scientifiques de niveau recherche, publiés ou non, émanant des établissements d'enseignement et de recherche français ou étrangers, des laboratoires publics ou privés. 


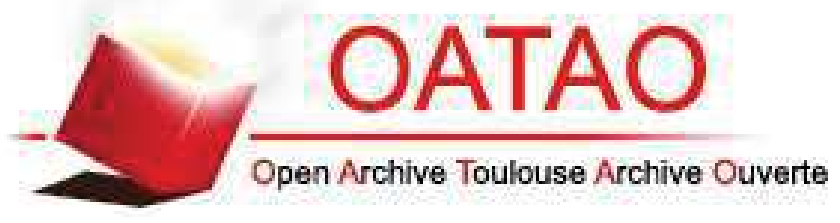

\section{Open Archive Toulouse Archive Ouverte (OATAO)}

OATAO is an open access repository that collects the work of some Toulouse researchers and makes it freely available over the web where possible.

This is an author's version published in: http://oatao.univ-toulouse.fr/22796

Official URL: https://doi.org/10.1108/IMDS-09-2016-0405

\section{To cite this version:}

Xu, Da and Karray, Mohamed Hedi and Archimède, Bernard A semantic-based decision support platform to assist products' eco-labeling process. (2017) Industrial Management \& Data Systems, 117 (7). 1340-1361.

Any correspondence concerning this service should be sent to the repository administrator: tech-oatao@listes-diff.inp-toulouse.fr 


\title{
A semantic based decision support platform to assist products' eco-labeling process
}

\begin{abstract}
With the rising concern of safety, health and environmental performance, eco-labeled product and service are becoming more and more popular. However, the long and complex process of eco-labeling sometimes demotivates manufacturers and service providers to be certificated. In this paper, we propose a decision support platform aiming at further improvement and acceleration of the eco-labeling process in order to democratize a broader application and certification of eco-labels, also to consolidate the credibility and validity of eco-labels. This decision support platform is based on a comprehensive knowledge base composed of various domain ontologies that are constructed according to an official eco-label criteria documentation. We present a reasoning methodology based on inference with SWRL (Semantic Web Rule Language) rules which allows decision making with explanation. Through standard RDF (Resource Description Framework) and OWL (Web Ontology Language) ontology query interface, the assets of the decision support platform will stimulate domain knowledge sharing and can be applied into other applications. A case study of laundry detergent eco-labeling process is also presented in this paper.
\end{abstract}

Keywords: Ontology Engineering, Decision Support, Eco-labeling, Knowledge Base, OWL, SWRL

\section{Introduction}

Since recent decades, there has been a growing demand from consumers, especially in developed economies, that products must be safe to use and are encouraged to do less harm to the environment. From a global point of view, promote of environment-friendly produce-consume-recycle progress will contribute not only to the life quality but also to the economy itself. The need of evaluating a product's safety, effectiveness and environmental performance has led to the establishment of eco-labels in order to certificate a product or service that meets certain environmental criteria.

On the other hand, for an eco-label applicant, usually a manufacturer or a service provider, it is easy to provide the required information in whatever formats. However, the difficulties encountered in the evaluating process are representative in decision-making process. To efficiently assess product or service, we need to manipulate different types of voluminous data; take into account different criteria and conduct a multi-criteria analysis; consider different phases of product or service life cycle. Usually, a group of human experts coming from various domains will work together and the evaluating process will take a long time, and errors and conflicts may exist. In addition, the evaluation result is actually a good resource that could have been made better use of. In order to better solve problems above, we propose to design and develop a decision support platform to assist the eco-labeling decision making process.

According to the survey made in (Golden et al., 2010), among single-standard eco-labels, the most common labels for time required to certification was three to six months, with $37 \%$ of respondents falling into this category. The average 
time to certification across single-standard labels is 4.33 months, the standard deviation is 4.37 months, which indicates that the time to get certificated is quite long, especially for some SMEs (Small and Medium-sized enterprises) and there is still a significant lack of uniformity in the market. Some digitalized management software tools are currently used, however, the evaluation process of eco-labeling certification is still mainly accomplished by the manual inspection or checking of domain experts. If some decision support tool is used in the heavy-duty and knowledge-intensive evaluation process, we believe that the response time will be drastically shortened and the certification cost will be reduced. This is the main gap we try to fill in this paper. We believe that faster certification with quality and credibility means more opportunities for enterprises and more efficient market. This will also be helpful when eco-labeling is introduced to a fast developing and much bigger market i.e. China and India.

Our research is to develop a decision support platform in the scope of eco-label certification i.e. eco-labeling process. The decision support process will take target product's profile as input, compare it with criteria which are stored in the knowledge base composed of ontologies, then generate the reasoning result and argumentation or explanation that tells whether the target product can be eco-labeled or not. A significant improvement of our approach compared to traditional decision support systems as mentioned and discussed in (Whyte, 1986) and (Eom, 1999) lies in the way the knowledge and data is stored. The knowledge cross-covered in such a decision support process will be represented in modularized ontologies and stored in a knowledge base. Synthetic engineering methods are proposed to organize these modularized ontologies in configurable context by combining them with infrastructure based on E-Connection(Grau et al., 2006). Another advantage of our approach is the argumentation or explanation that accompanies the labeling decision. In the light of the argumentation, decision makers can have clearer understanding on how the decision result is made and why. As is illustrated on the left side of Figure 1, the objective of our research is to assist and accelerate the evaluation process of eco-labeling to help domain experts make wiser decisions on behalf of the administration and management of eco-labeling. The proposed knowledge base of this system will contribute the reuse of eco-label products knowledge and improve its interoperability with other systems, such as EMS (Environment Management Systems), PLM (Product Lifecycle Management) systems, and ERP (Enterprise Resource Planning) systems. Simultaneously, from the point of view of a producer as an eco-label applicant, such a decision support tool can serve as a simulation tool that will assist the design and validation phases of new product's development, as shown on the right side of Figure 1.

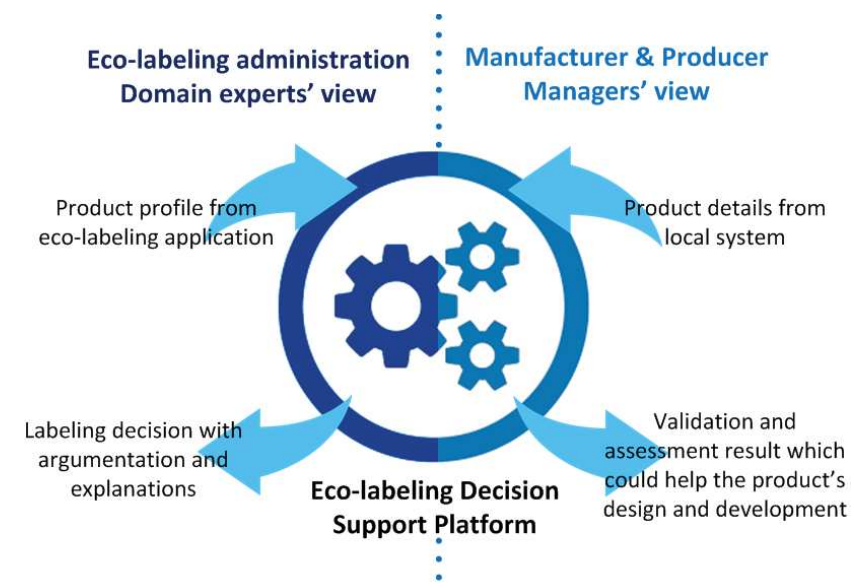

Figure 1: The objective and function of the decision support platform from both eco-labeling administration and producer's point of view.

The structure of this paper will be as follow: First, in the State of art section, we will introduce briefly what is eco-label, what is EU Eco-label and the current eco-labeling decision support process. We will also give a brief review 
of ontology, modular ontology and relative application (especially in decision support). In section 3, mechanism of the decision support process will be explained in details. Section 4 presents a preliminary software infrastructure implementation, and a case study of laundry detergent product group. In Section 5, we will have some discussion about the system, its limitation and our future work plan. At last, we have the conclusion section.

\section{State of art}

\subsection{Eco-label, EU Eco-label and Eco-labeling process}

According to Global Eco-labelling Network (GEN) ${ }^{1}$, "eco-labeling" is a voluntary method of environmental performance certification and labelling that is practiced around the world. An "eco-label" is a label which identifies overall, proven environmental preference of a product or service within a specific product/service category. They usually concern the whole life cycle of the product and are issued by third party (Lefébure and Rosales Muñoz, 2011). The International Organization for Standardization (ISO) has identified three broad types of voluntary labels, with most eco-labels fitting under the Type I designation (Global Ecolabelling Network: What is ecolabelling?, 2016).

Eco-labeling has numbers of benefits from various points of view. First, eco-labeling is a good way to inform consumers of selected products' environmental impacts. In the practice of some existent eco-labeling, the fitness of use and human health aspects are also included. Eco-labeling is generally cheaper than regulatory controls. By empowering customers and manufacturers to make environmentally supportive decisions, the need for regulation is kept to a minimum. This is beneficial to both government and industry (Vilhelmiina Ihamäki, 2014). Eco-labeling will also stimulate market development and encourage continuous improvement on product and service. From global point of view, at present, according to Ecolabelindex.com ${ }^{2}$ there are over 450 Eco-labels in 197 countries, and 25 industry sectors. In the summary and report of Global Ecolabelling Network (GEN) in $2014^{3}$, certificated products worldwide exceeds 250,000. As indicated in the "2010 Global Ecolabel Monitor" survey ${ }^{4}$, most surveyed eco-labels are operated in Europe and North America and the majority of them are third-party certification systems.

As a quite successful example among all the eco-labels, EU Eco-label is chosen to be our concrete study case. Created in 1992, the EU Eco-label is the only official European ecological label authorized for use in every member country of the European Union (EU Eco-label scheme - What is it?, 2016). Until 2011, there are over 1300 enterprises that have been issued EU Eco-label licenses. By September of 2014, there are already over 43,000 products or services being labelled (Facts and Figures - Ecolabel - European Commission, 2016). France is always an important contributer to EU Eco-labeling. By March of 2016, 486 enterprises in France have EU Eco-label licenses in various product groups and that makes France the first place as for the enterprises' possession of EU Eco-label licenses. As illustrated in Figure 2, the remove of certain product or service group indicates that the validation and alteration of EU Eco-label criteria is continuous and up-to-date. It also implies the change of knowledge and rules.

\footnotetext{
${ }^{1}$ http://www.globalecolabelling.net/

${ }^{2}$ http://www.ecolabelindex.com/

${ }^{3}$ https://www.flipsnack.com/FA69B9F569B/global-ecolabelling-network-agm-2014-report.html

${ }^{4}$ http://www.wri.org/publication/2010-global-ecolabel-monitor
} 


\section{TOTAL EU ECOLABEL LICENCES PER PRODUCT/SERVICE GROUP}

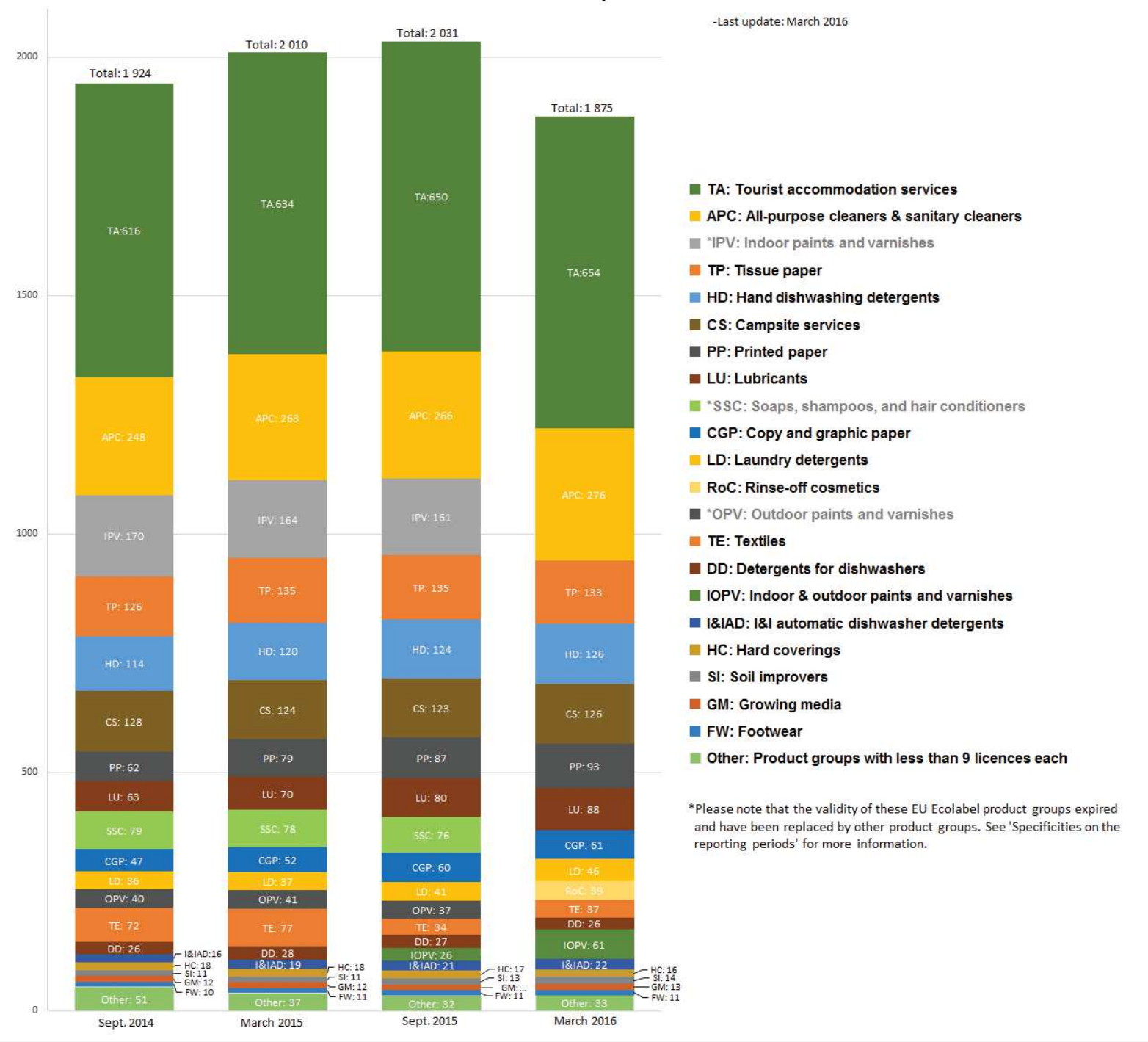

Figure 2: Total EU Eco-label licences per product/service group

\subsection{Eco-labeling decision support and ontology application}

Although some environmental performance assessment methods and tools have been developed, e.g. LCA (Life Cycle Assessment), ERA (Environmental Risk Assessment) and LCC (Life Cycle Cost) mentioned in (Curran et al., 2005), EDIP 1997 (Dreyer et al., 2003), OMNIITOX (Molander et al., 2004), IMPACT 2002+ (Jolliet et al., 2003), etc, most of these methods and tools focus barely on technical analysis. There is a lack of appropriate software implementation support especially in terms of decision support functionality. In the work of (Houe and Grabot, 2007), a tool to verify the compliance of a product with given norms and standards in terms of the recyclability of industrial products is described. It is shown how the knowledge contained in eco-labeling standards and norms in textual form can be translated into constraints. NIAM/ORM (Object-role Modeling) can be used for formalizing the product data and the verification of the eco-label compliance turns into a CSP (Constraint Satisfaction Problem). In their later work in (Houe and Grabot, 2009), the authors present how CLAIRE (Combining Logical Assertions, Inheritance, Relations 
and Entities) language is used to solve the CSP. Formalizing the data and knowledge contained in textual materials into rules is great, but their work serves only to the design phase of the product. The formalism they chose and the CSP translation are too specific to be reused and hard to interoperate with other systems. In (Andersson et al., 2012), the authors present and discuss the implementation of product life cycle assessment for eco-labeling using DES (Discrete Event Simulation). CMSD (Core Manufacturing Simulation Data)(Leong et al., 2006) is used to facilitate the definition of manufacturing information related to production operations in order to address interoperability issues between simulations and other manufacturing applications. But this work is dedicated to type III eco-labels exclusively. In (Minhas and Berger, 2014), development of environmental knowledge management tool capable of providing planners and production managers the knowledge related to the potential environmental impact of the manufacturing choices in a distributed automotive manufacturing scenario is presented. A web-based software solution is developed for customized user query interface, and ontology based knowledge-base is implemented and validated. However, the explanation function or argumentation of the result of aforementioned systems are weak or absent.

Ontology and Semantic Web technologies have been used in DSS (Decision Support System) during the past decade to solve a number of different tasks, such as information integration and sharing, web service annotation and discovery, and knowledge representation and reasoning (Blomqvist, 2014). However, according to our survey, there is still no software tools that implement eco-labeling decision support by means of inter-connected ontologies, SWRL rules' reasoning and explanation. Our work explores new possibility of ontology application and its engineering practicability. Reasoning based on SWRL rules, especially, is the core mechanism of our decision support process. We expect that our research can be of some help and inspiration to the application of Semantic Web technologies and the assets of our research, especially the ontology knowledge base, could be exploited by other eco-labels and reused in other similar application or systems.

\subsection{Ontology and modularized ontology}

In Computer Science, we refer to an ontology as a special kind of information object or computational artifact (Guarino et al., 2009). Studer et al. (Studer et al., 1998) gave definition stating that: "An ontology is a formal, explicit specification of a shared conceptualization." Today, so many ontologies and knowledge repositories have been developed and adapted into applications, especially in biomedical domains (Pathak et al., 2009). Lots of problems are encountered when knowledge engineers and general users want to understand and reuse the ontologies into their own software development. One reason of such difficulties is the semantic confusion between domains. In application of ontology, there is also definite need to reuse knowledge from multiple remote ontological sources. That partly explains why ontology is supposed to be modular. It is known that, when knowledge is distributed, the idea to collect all knowledge into a single repository (i.e. the integration approach) is very difficult to implement, because of semantic heterogeneity calling for human processing(Parent and Spaccapietra, 2009).

Ontology modularization or modular ontology is an interesting approach that deals with ontology reusability. In general, there are two important aspects of ontology modularization: independently developing modules that can be integrated coherently and uniformly (ontology composition) or extracting such modules from an integrated ontology for supporting a particular use case(ontology decomposition) (Pathak et al., 2009). Our research focuses on the first aspect and we emphasize more on the reuse of ontologies and interoperability of ontology knowledge base. To define and specify certain modular ontology, several popular formalisms are proposed, i.g. E-Connection(Grau et al., 2009), Distributed Description Logics (DDL) framework(Serafini and Tamilin, 2009), DRAGO(Distributed Reasoning Architecture for a Galaxy of Ontologies)(Serafini and Tamilin, 2005), and Package-Based Description Logics(Bao et al., 
2009). An E-Connected ontology contains information about classes, properties and their instances, but also about a new kind of properties, called Link Properties, which establish the connection between the ontologies. Our ontology knowledge base that supports the eco-labeling decision support process borrows the idea of E-Connection, because we think that E-Connection has a relative easy syntax and semantics schema which allows a very high possibility for modules to be inter-connected in large scale. There is a brief illustration of the modularized design of the ontology knowledge base in Section 4.

Much effort has been done in terms of ontology application in industry and quite amount of ontologies in different formalisms and various domains have been developed. While, we still have not mature methodologies for ontology engineering in various scenarios, neither well-developed support tools. The number of good practice and business success that take advantage of ontology engineering and Semantic Web technologies is still very limited. The vision of Semantic Web proposed by (Berners-Lee et al., 2006) is far yet reached. When it comes to Eco-labeling, despite much domain knowledge as criteria or standards have been established. However, they are not very well linked and exploited yet. As indicated in the "2010 Global Eco-label Monitor" report, less than 30\% of eco-labels recognize or are recognized by other labeling organisations, indicating an opportunity for increased collaboration amongst eco-labels to reduce confusion amongst users of the eco-labels. With nearly all eco-labeling organisations requiring some form of metrics reporting, there is further opportunity to collaborate and create more aligned standards. Faced with such challenge, we believe that the interoperable and sharing nature of ontology should facilitate eco-labeling criteria or standards' mapping and collaboration.

\section{An decision support approach based on ontology knowledge base}

\subsection{An overview of the decision support process}

Figure 3 presents a simplified outline of the eco-labeling decision support process from two different points of view in terms of the eco-labeling administration's domain experts (scenario 1) and the producer \& service provider (scenario 2). There are three roles as participants involved in the decision making process: the applicant as manufacturer or service provider who initiates the eco-label application; the domain experts who take the results of the system and make the final decision on whether the product or service is qualified to be eco-labeled or not; and the member country's authorized Competent Body who is responsible for providing guideline and advice to the applicant while the applicant prepares the required product or service's information. 

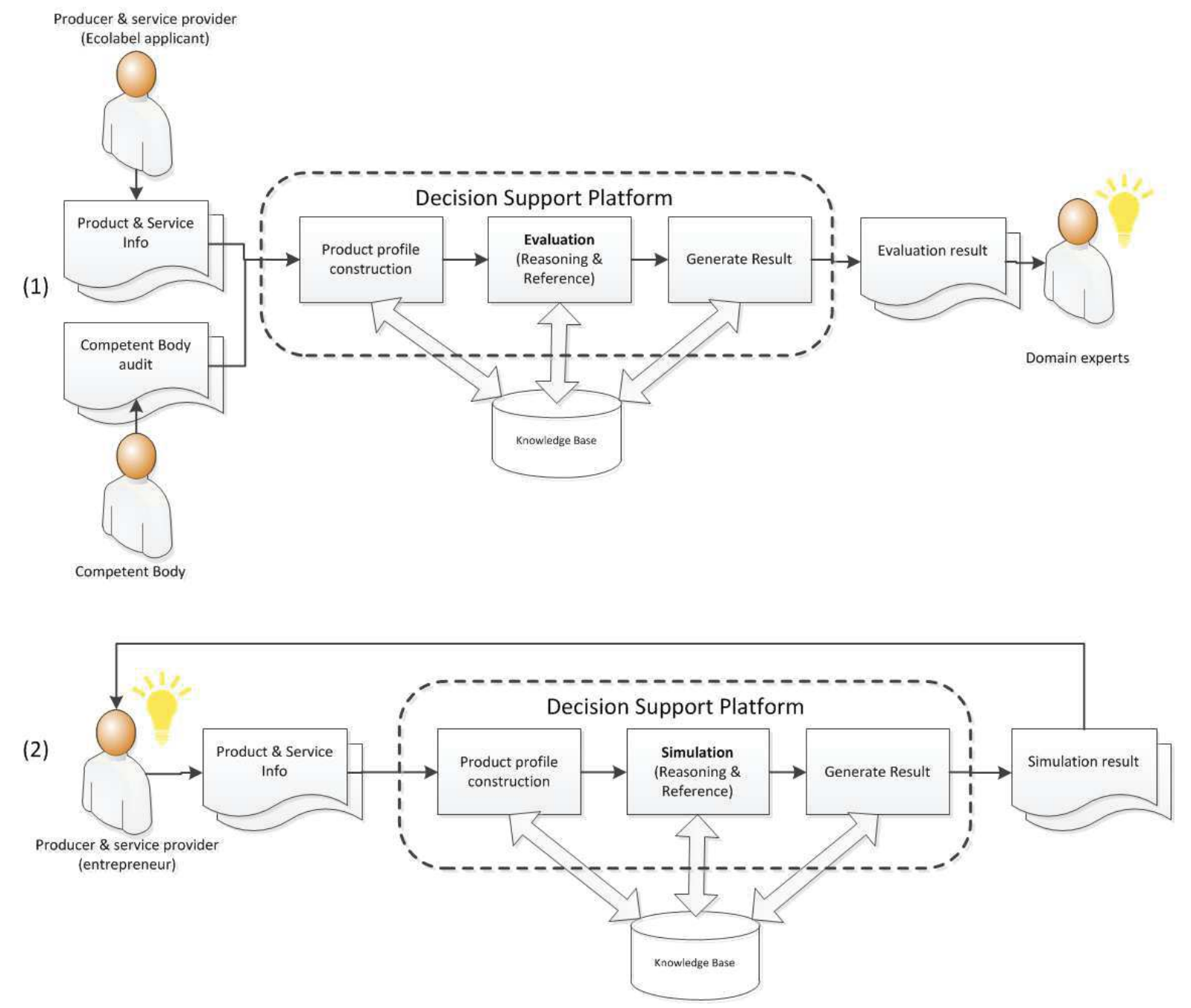

Figure 3: Eco-labeling decision support process from two different points of view in terms of the eco-labeling administration's domain experts (1) and the producer \& service provider (2)

In a standard evaluation or simulation process, a detailed description of product or service is provided as the key input of the system. The platform retrieves concerned information from the product description and builds a machine readable structured document. Then, the structured document will be transferred into a Product Profile Ontology (Abox) in accordance with an Template Ontology (Tbox) retrieved from the ontology knowledge base. Afterwards, the system will select related domain ontologies from the knowledge base. Towards these ontologies, a modularization and refinement formation will proceed in order to gather the very necessary knowledge parts (usually the obligatory criteria rules) to build a merged Criteria Ontology. In the next step, the inference takes place on the combination of Product Profile Ontology and Criteria Ontology, verifying if the Product Profile Ontology that contains description of the product comply with the corresponding criteria. At last, an argumentation generation component will parse and translate the conflicts between Product Profile Ontology and Criterion Ontology so as to generate the final report for human experts review. All the document processing, reasoning or generating process will be supported by a comprehensive knowledge base composed of ontology. 


\subsection{Architecture and dynamics of the decision support platform}

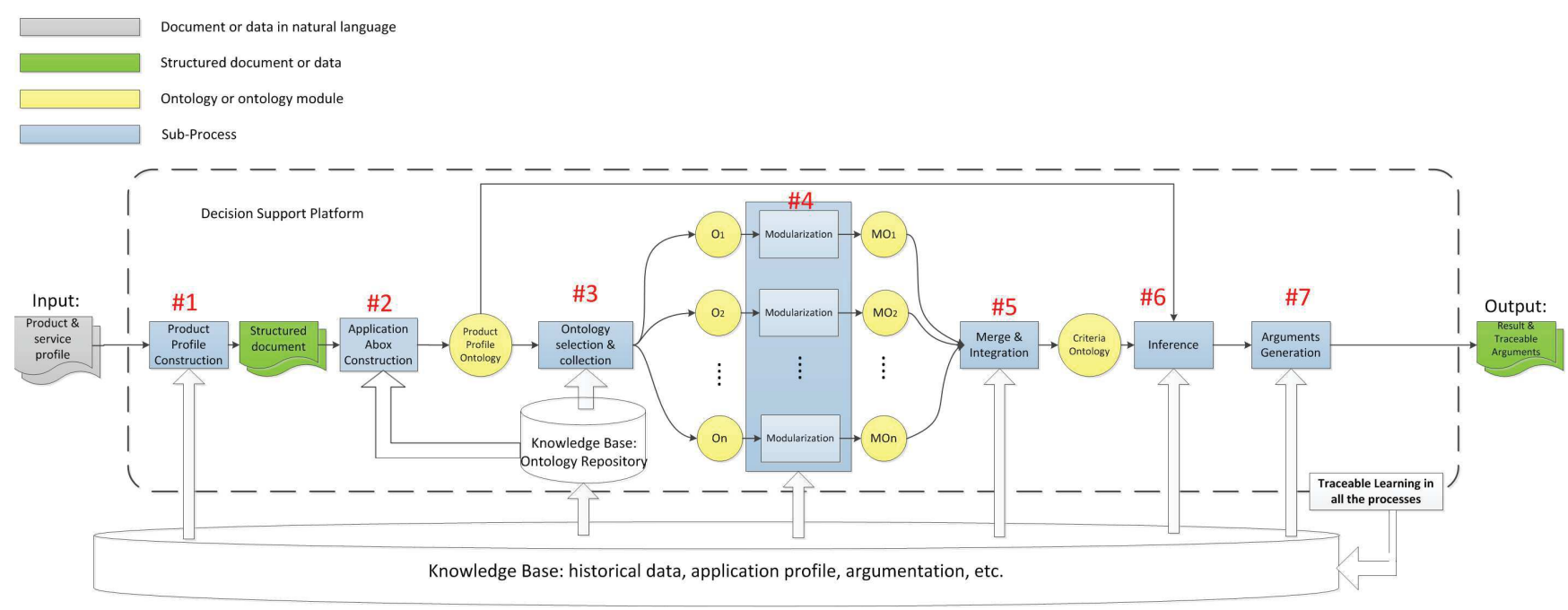

Figure 4: Functioning of the decision support platform for Eco-labeling

Figure 4 is a detailed functioning of the eco-labeling decision support platform. The rest part of this subsection is devoted to describe how we make use of the knowledge base to facilitate a product or service evaluation with traceable argumentation.

Sub-process 1: Product profile construction This is the very first step of the decision support process and the detailed product or service's profile is required. The inputs are the documents provided by the applicant and the output is structured document in XML. For now, GUI of electronic forms are used to capture product's profile. The information provided by applicant contains not only numeric parameters and data but also product packaging artwork, administrative or legislation declaration, laboratory analysis report. Some are easy to be processed by machine such as all kinds of forms, while some other need human reading and comprehension. 

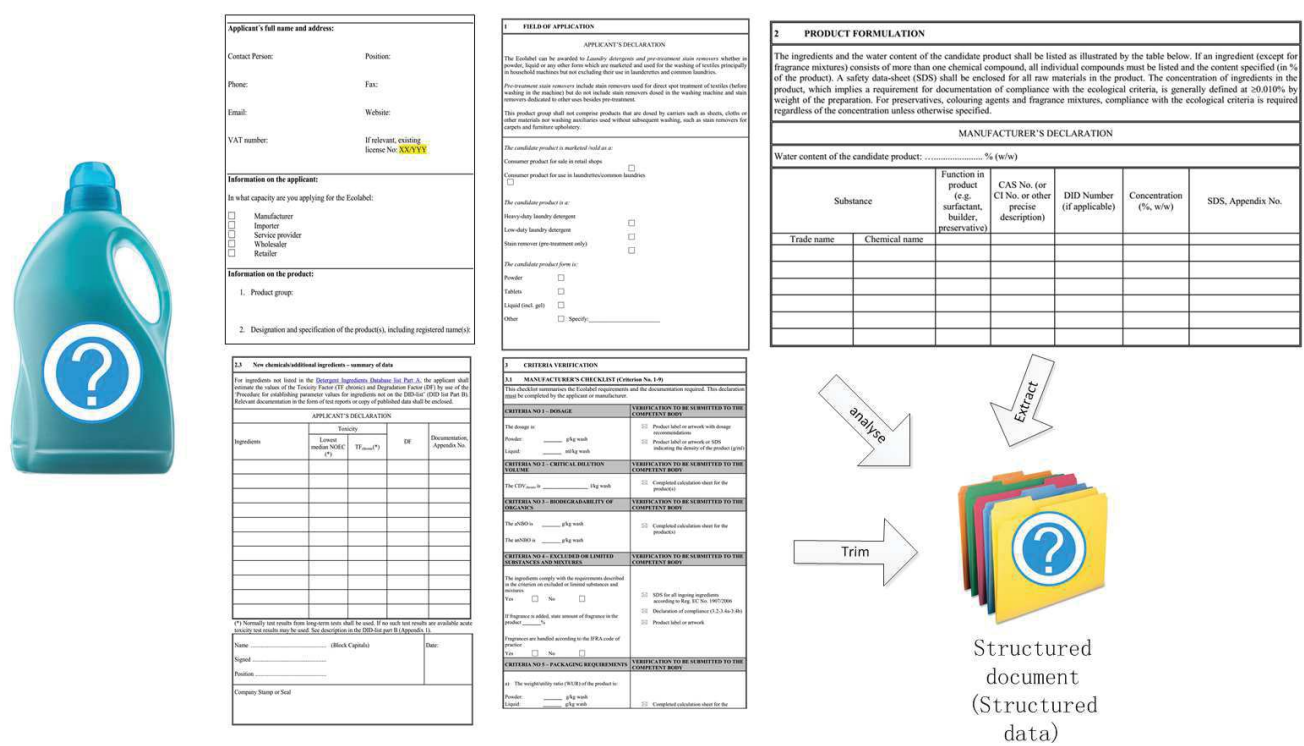

Figure 5: Sub-process 1: Product profile construction. In this step, useful product information will be extracted from product profile documentation and stored into a structured format.

Sub-process 2: Product Profile Ontology Construction This step is very critical to the subsequent steps. As the knowledge base is composed of ontologies of different product groups, we need to choose and transfer or translate the input structured product profile into corresponding ontology assertions. In this translating process, a Template Ontology is required. Template Ontologies are predefined in the ontology repository which is also a part of the knowledge base. A product profile will notify the ontology repository to query for the right template. A Template Ontology is like a stereotype container defined in ontology language. It contains information about date fields, their formats and other information which are required by the decision support platform. Usually a Template Ontology is a Tbox, in which few instance or individual exist, it mainly specifies the conceptualization of a product or service. Followed by the structure of the template or stereotype, the product \& service information will be organized and transferred into an Abox called Product Profile Ontology which contains a bunches of individuals.

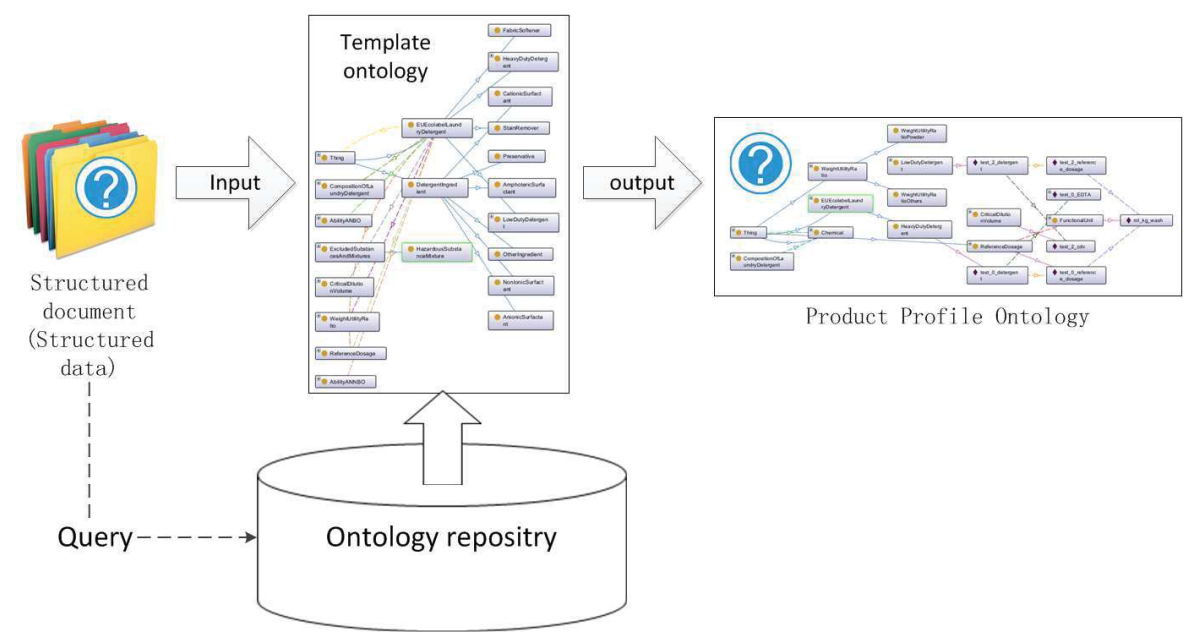

Figure 6: Sub-process 2: Product Profile Ontology (Abox) Construction. A Template Ontology (Tbox) corresponding to the product type is retrieved from the ontology repository, then an Product Profile Ontology is constructed. 
Sub-process 3, 4, and 5: Criteria Ontology generation The objective and output of sub-process 3, 4, and 5 is to generate the Criteria Ontology. Based on the semantics of input product \& service profile in Product Profile Ontology, ontology or ontology modules will be selected and collected from the knowledge base and the ontology repository. We call this integration of modules as Criteria Ontology, an knowledge component which holds product criteria. Please note the difference between Product Profile Ontology and Criteria Ontology. The former one is mostly a descriptive assertion and instantiation that records the product or service's profile, while a Criteria Ontology is supposed to contain the assessment criteria and guidelines. It is in compliance with the official eco-labeling criteria documents. In a technical point of view, all the SWRL rules should be defined in the Criteria Ontology.

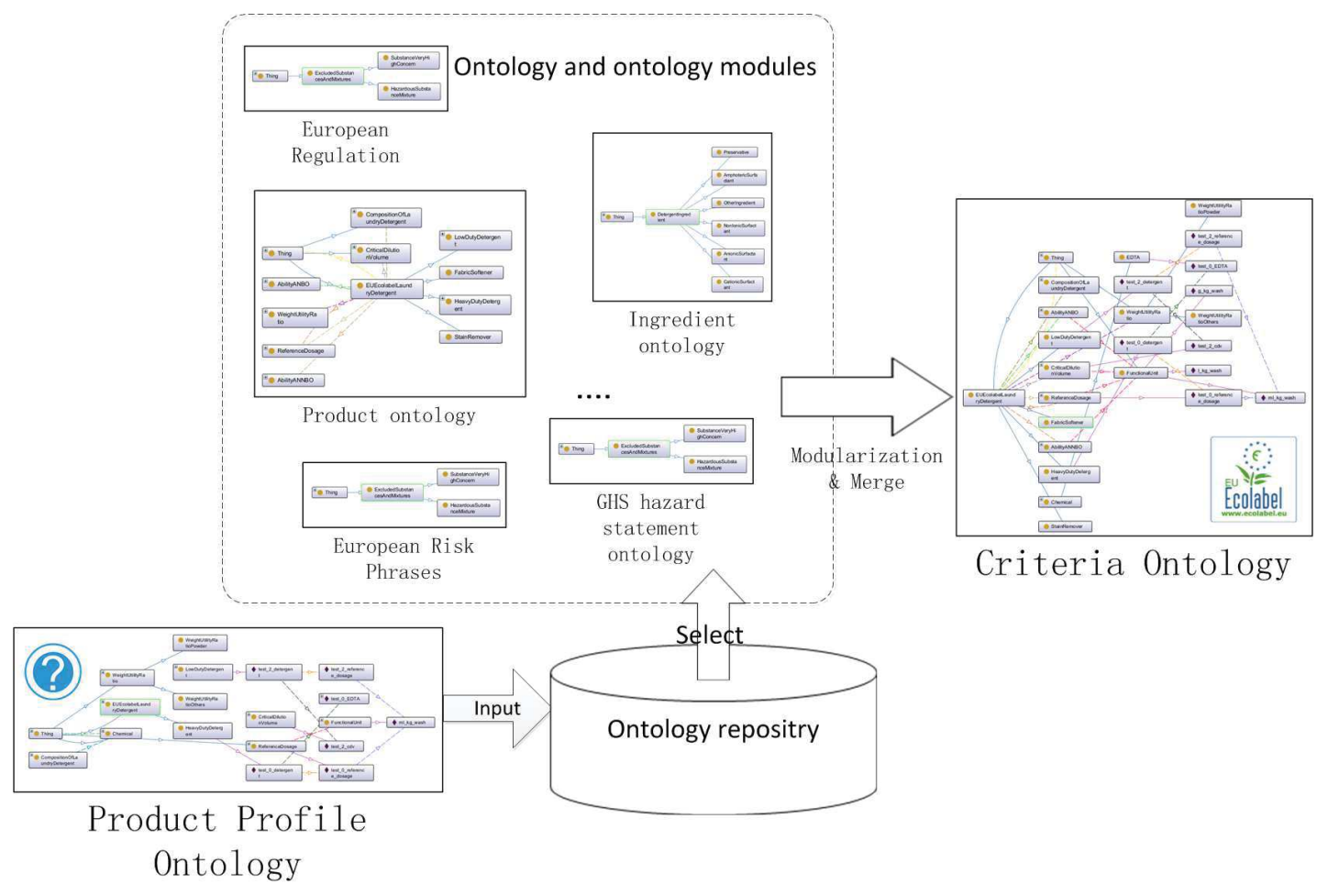

Figure 7: Through sub-process 3 to 5, a Criteria Ontology is constructed.

In sub-process 4, modularization may be applied on the selected ontologies or modules. Because these selected items could be quite complex and big in consideration of their volume and quantity. If we combine these components together directly, unnecessary knowledge and information would exist in the Criteria Ontology and that will introduce negative influence to the reasoning performance.

In sub-process 5, a merge and ontology integration is proceeded to finalize and generate the Criteria Ontology.

Sub-process 6 and 7: Inference \& Arguments generation Taking the input Product Profile Ontology and the Criteria Ontology, inference sub-process takes charge of indicating which part in the Product Profile Ontology doesn't comply with the rules defined in the Criteria Ontology. Then, arguments generation component will generate reasons and explanations on why these inconsistency and noncompliance exist. In addition to the direct inference result in ontology language, the arguments generation component will parse and translate the reasons and explanations into a reading friendly output report for human experts' review. Till here the task of decision support system is finished and the following procedure will be up to the experts to judge the results and feedback to the applicant. 


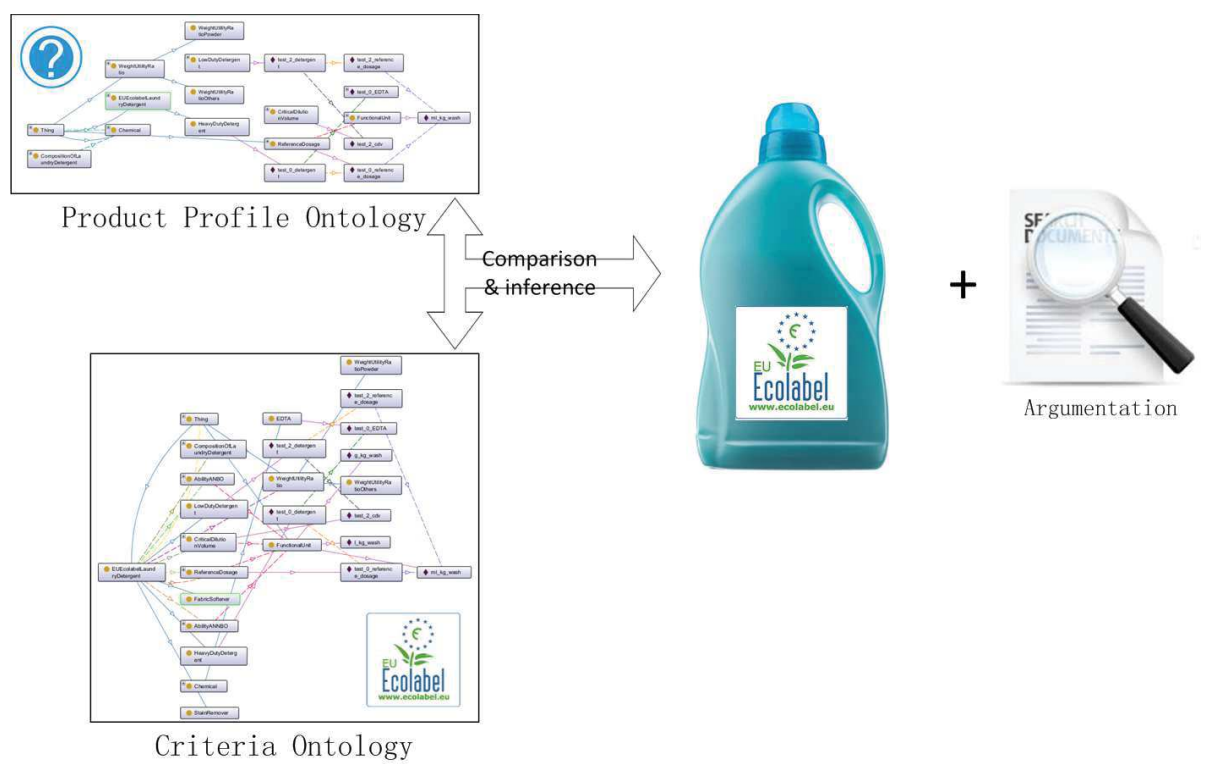

Figure 8: Via the comparison and reasoning applied between the Product Profile Ontology and Criteria Ontology, eco-labeling suggestion and argumentation are obtained at last.

To finish this section, note that all the sub-processes will be supported by the knowledge base. The knowledge base preserves comprehensive types of data and knowledge. A critical part of the knowledge base is the ontology repository(see Figure4), where all the Template Ontologies, Product Profile Ontologies, Criteria Ontologies are kept. The rest part is designed to store all the historical data, application, reasoning result, and argumentation generated during every decision support process. The knowledge base is connected to other data sources locally or remotely. To achieve a better interoperability performance, the ontology repository is equipped with public semantic data accessing interface, which allows the ontology and data stored locally to be accessed by other applications. In the opposite direction, the knowledge base is designed to be able to browse other knowledge base or ontology repository e.g. $\mathrm{ChEBI}^{5}, \mathrm{ChEMBL}^{6}$, BioPortal $^{7}$ and PubChem ${ }^{8}$ to acquire extra information. With such an open information sharing mechanism, it is guaranteed that the knowledge base can be shared. This will be the cornerstone of interoperability when the decision support system is about to cooperate with other systems or is to be integrated into other systems, such as PLM system or EMS systems.

\section{Infrastructure implementation and case study}

\subsection{Case study of EU Eco-labeling on laundry detergent}

As we've seen from the state of art section, the EU Eco-label product catalog covers more than thirty product groups which is a quite big criteria system. For our primary implementation, we start from single product group to test and validate our decision support process. In this paper, laundry detergent product group is chosen to be our study case. Because the criteria volume of laundry detergent is neither too big nor too small. In the market, quite a number of

https://www.ebi.ac.uk/chebi/init.do

${ }^{6}$ http://www.ebi.ac.uk/rdf/services/chembl/sparql

${ }^{7}$ http://bioportal.bioontology.org/

${ }^{8}$ https://pubchem.ncbi.nlm.nih.gov/search/\#collection=compounds 
laundry detergent products are already eco-labeled and such a popularity can provide enough successful eco-labeling application cases to be further studied.

A necessary part of the implementation of the decision support platform infrastructure is the knowledge base. For our laundry detergent case, a modularized domain ontology is constructed. As illustrated in Figure 9, this modularized scheme separates "entities" and "rules". On the left side, the modules with solid border line represent "entities". The modules with dashed border line represent the "rules". The "entities" are the concepts and initial instances referred in the laundry detergent eco-labeling criteria. They are used to describe the conceptualization of the laundry detergent world in the scope of eco-labeling. While, the "rules" are the criterion that reflect people's intention and constraints imposed to the world. This modular design has advantage in terms of reuse and change of the ontology. Since the descriptive part of the ontology does not change very much but the criterion or the "rules" are altered from time to time, once the "entities" and "rules" are separately managed, we can identify one criterion module rapidly and do the updates without disturbing the other modules(Da et al., 2016).

This domain ontology in OWL is constructed by using the popular ontology editing tool Protégé. Most of the "rules" modules (the modules with dashed line border in Figure 9) are developed in SWRL. By using SWRL, general reasoners can be launched to do the inference on the Product Profile Ontology and generate the argumentation, which is the essential task of the decision support platform. Much more details will be elaborated in Section 4.3.

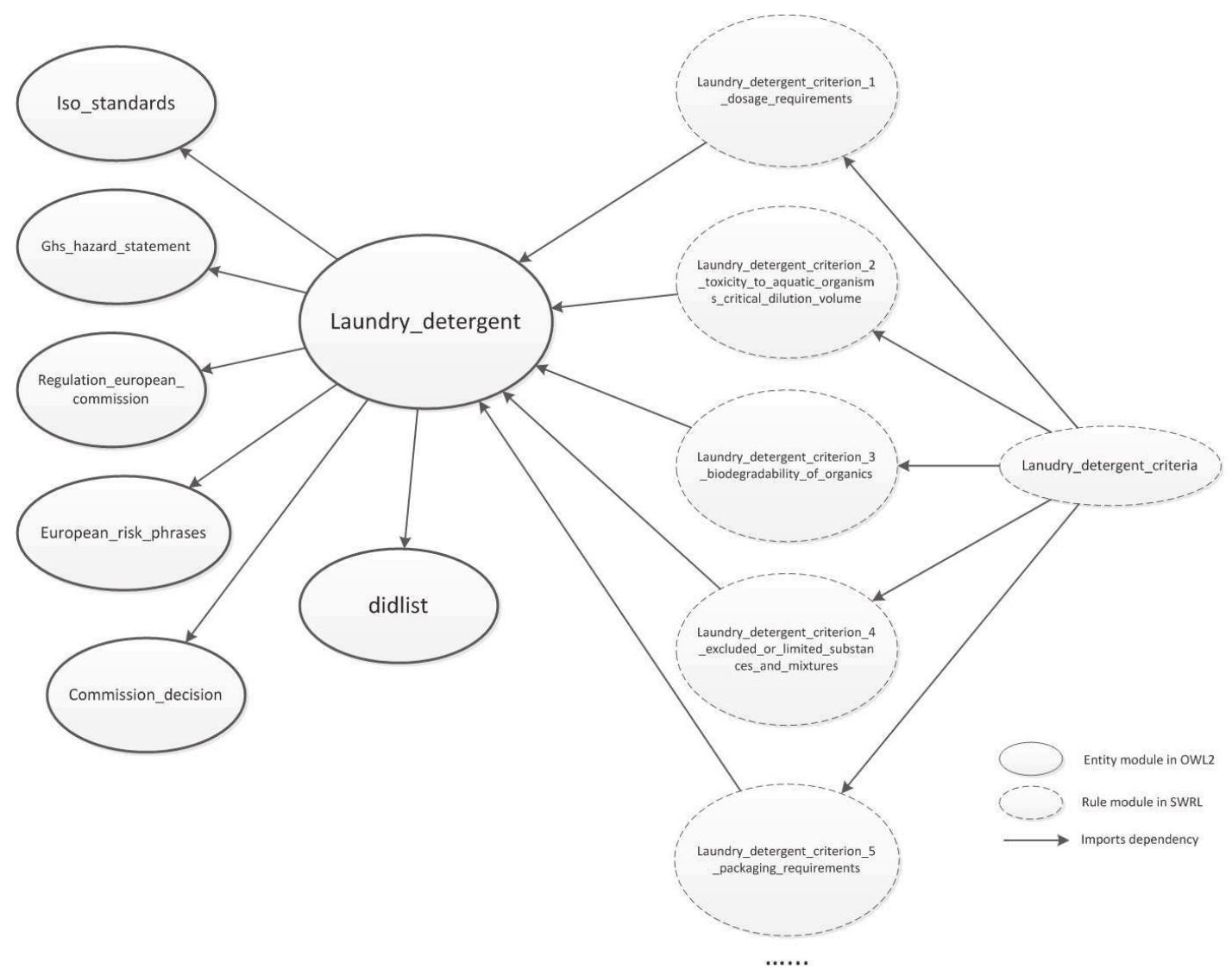

Figure 9: Modularized ontology knowledge base for laundry detergent product's eco-labeling criteria 


\subsection{A preliminary infrastructure implementation}

To start an application, it is suggested by EU Eco-label to use an on-line application management tool called $E C A T_{-} A d \min ^{9}$. First of all, the applicant should register itself as a company, then a detailed product or service profile should be submitted. However, the information being collected here is no more than some basic registry information about the applicant or the enterprise. This on-line tool will facilitate the routine work of application management on the level of EU Commission, but not the pivotal and actual product evaluation part. As for the evaluation, detailed parameters, description as well as test reports of the product or service are needed. However, The decision making part is not involved in this ECAT_Admin tool. Figure 10 is document snapshot taken from the "Application form and guidance document for laundry detergents". An applicant must fill such kind of forms, and send them to the competent body. For the evaluation and assessment process, there is no software tool support.

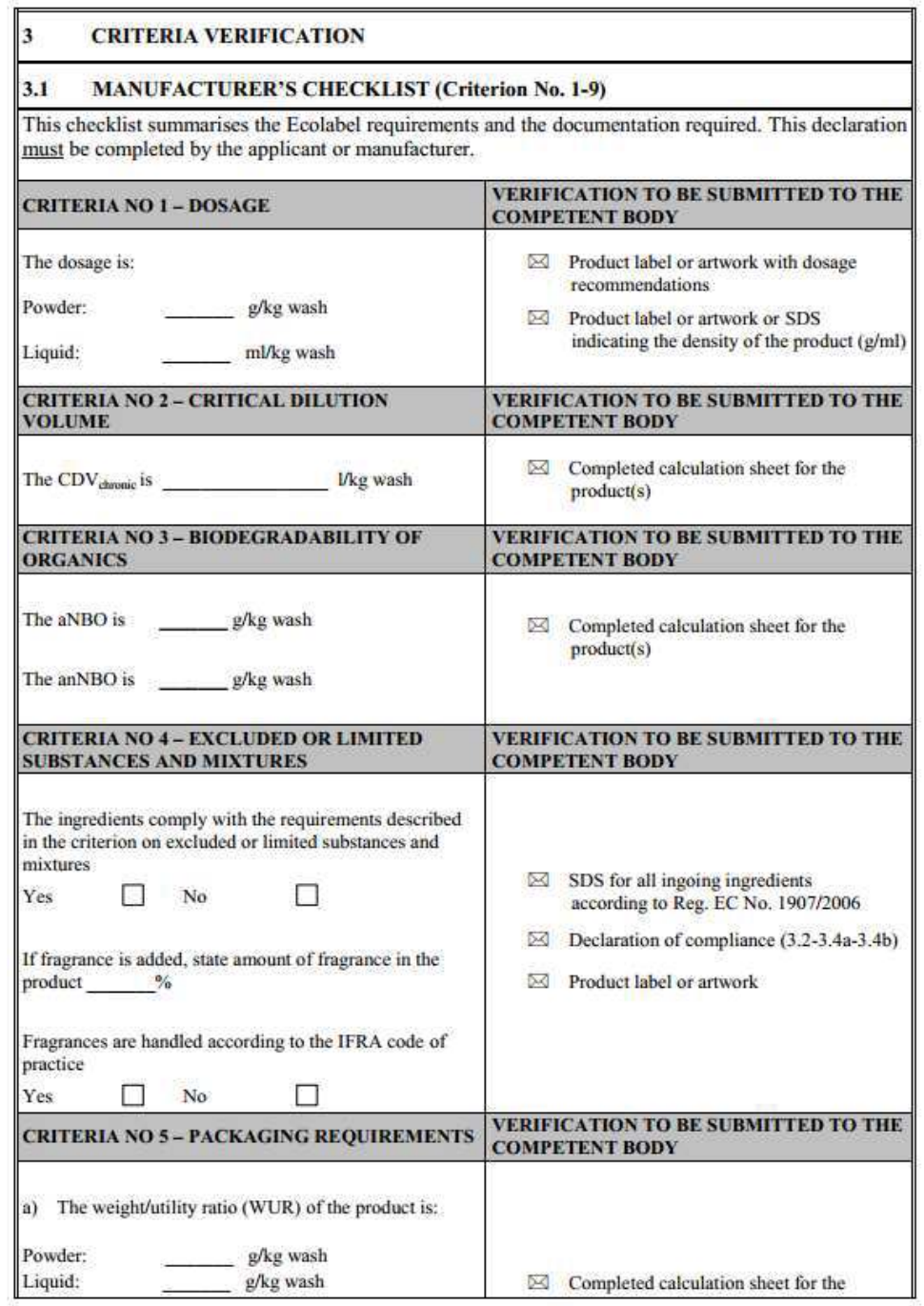

Figure 10: Application form example: detergent product parameter verification check-list for each product criterion.

As a primary step, in this work, we develop a prototype decision support system as illustrated in Figure 11. This prototype software serves mainly to the domain experts and decision makers who work for the eco-labeling. Before an

${ }^{9}$ https://webgate.ec.europa.eu/ecat_admin/ 
applicant submit their product or service profile, he can also use such kind of tool to do some preparatory test and simulation. In this prototype system, we have each application stored in a file of certain format. The user loads the application file and a tree structure will show up on the left side to allow the user to navigate between parts of the application. Before the eco-labeling decision support process is started, the system will retrieve the product profile stored in the file, combine it with the corresponding product Template Ontology to build a Product Profile Ontology. (In our prototype system, the default product Template Ontology is the laundry detergent). The panel on the right side shows details of different parts of the application. The console panel at the bottom is used to display feedback and reasoning results.

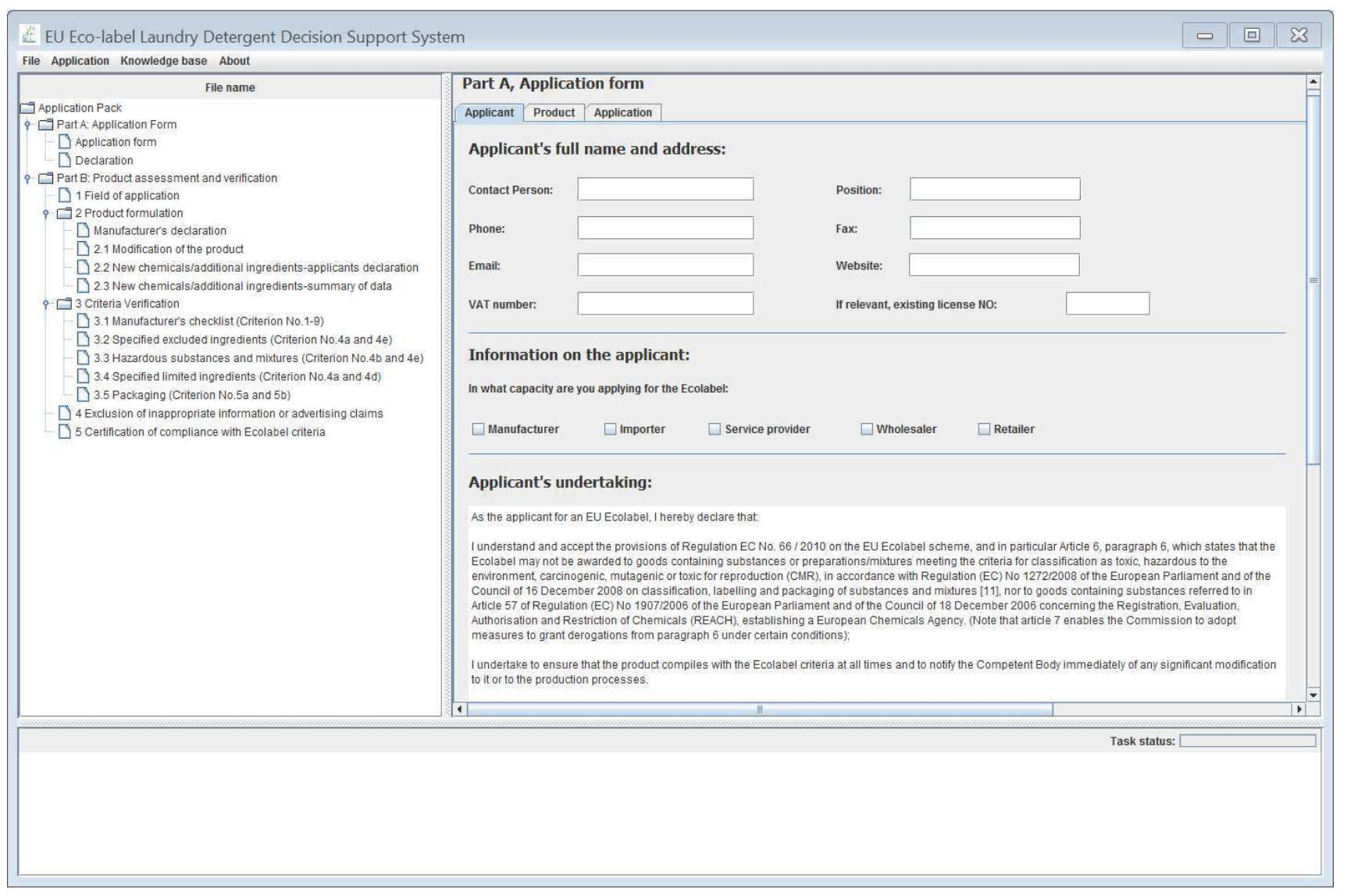

Figure 11: A Java prototype system for the Eco-labeling decision support platform.

The prototype system also provides a query portal which allows users or other applications to launch standard SPARQL query to access the ontology knowledge base. This functionality is implemented with the Jena API ${ }^{10}$, we are trying to publish this query portal on the web. Figure 12 is the interface for this query portal and the query result will be displayed in the console panel in Figure 11.

${ }^{10}$ https://jena.apache.org/ 


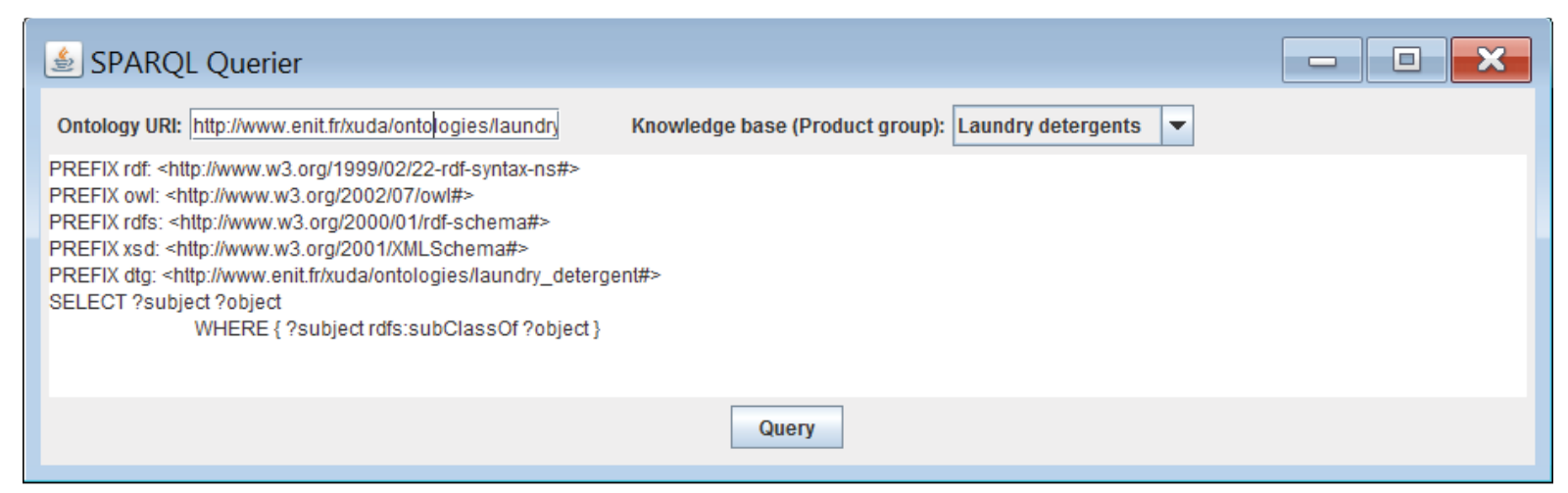

Figure 12: A SPARQL query portal of the prototype system.

\subsection{Reasoning and argumentation}

An considerable advantage of using OWL ontology is that the underlying DL (Description Logic) formalism allows reasoning. Actually the DL computation complexity and the development of reasoners are very important research issues for ontology and ontology engineering. Investigating the trade-off between the expressivity of DLs and the complexity of their inference problems has been one of the most important issues in DL research(Baader and Sattler, 2001). As for the expressiveness of OWL, it's mostly related to the underlying DL expressiveness. Concerning the latest W3C recommendation, there are three OWL schemes on different levels of expressiveness: OWL-Lite, OWL-DL, OWL-Full(McGuinness et al., 2004). OWL-DL is best supported by reasoners because it is decidable which means a proper trade-off between reasoning performance and expressivity. One famous algorithm implemented by many modern DL reasoners is called Tableau Algorithm.(Baader and Sattler, 2001) In this section, we focus on how to apply reasoner to do the reasoning and how to generate the argumentation. We will see how sub-process 6 and 7 are implemented in the prototype system of our decision support platform.

In Protégé editor, several third-party reasoners exist as plug-ins. In fact, today's reasoners can also stand along as APIs or even independent tools. Since Protégé is an open source project, for almost all its reasoner plug-ins we can find APIs that can be integrated into programming language like Java or $\mathrm{C}++$. Among the original reasoners carried along by Protégé (the version we used is Protégé 5.0.0 beta 24), FaCT ++ is a sound and complete reasoner for SHOIQ (the same description logic underlying OWL-DL)(Tsarkov and Horrocks, 2006). Pellet is also a sound and complete reasoner which would be very interesting for our research(Sirin et al., 2007). Hermit(Shearer et al., 2008) works best with our ontology knowledge base as for the SWRL rules, so all the reasoning tasks involved in this paper is completed by Hermit.

After checking all the criterion in the laundry detergent product, we translate them into SWRL rules. Take the weight/utility ratio part of packaging requirements criterion for example. This part of the criterion, which is quite simple, is to control the weight/utility ratio(WUR) of the product in order to use less packing materials to contain more products. In EU Eco-label criteria document, the details of this criterion is shown in Table 1.

Based on our criteria ontology, this criterion can be translated to SWRL rules like this:

1. CandidateLaundryDetergent(?a), hasDetergentProductType(?a, powder $)$, hasWeightUtilityRatio(?a, ?wur $)$, hasValue(?wur,?wur_value), xsd : double[> "1.2" ^^xsd:double](?wur_value) $\rightarrow$ RejectedDetergent $(? a)$ 
Table 1: Criterion of packaging requirements. The weight/utility (WUR) of the product shall not exceed the following values.

\begin{tabular}{cc}
\hline Product type & WUR \\
\hline Powders & $1.2 \mathrm{~g} / \mathrm{kg}$ wash \\
Others (e.g. liquids, gels, tablets, capsules) & $1.5 \mathrm{~g} / \mathrm{kg}$ wash \\
\hline
\end{tabular}

2. CandidateLaundryDetergent(?a), hasDetergentProductType(?a, liquid), hasWeightUtilityRatio(?a,?wur), hasValue(?wur,?wur_value), xsd : double[> "1.2" ^^xsd:double](?wur_value) $\rightarrow$ RejectedDetergent $(? a)$

3. CandidateLaundryDetergent(?a), hasDetergentProductType(?a, gel), hasWeightUtilityRatio(?a,?wur $)$, hasValue(?wur,?wur_value), xsd:double[> "1.2" ^^xsd:double](?wur_value) $\rightarrow$ RejectedDetergent $(? a)$

4. CandidateLaundryDetergent(?a), hasDetergentProductType(?a, tablet), hasWeightUtilityRatio(?a, ?wur), hasValue(?wur,?wur_value), xsd :double[> "1.2" ^^xsd:double](?wur_value) $\rightarrow$ RejectedDetergent $(? a)$

5. CandidateLaundryDetergent(?a), hasDetergentProductType(?a, capsule), hasWeightUtilityRatio(?a, ?wur), hasValue(?wur,?wur_value), xsd : double[> "1.2" ^^xsd:double](?wur_value) $\rightarrow$ RejectedDetergent $(? a)$

The basic idea for almost all the criteria rules is by introducing concepts called "RejectedDetergent" and "CandidateLaundryDetergent" in the ontology. At the start of the decision support process, we input product profile as instance of the "CandidateLaundryDetergent", we still don't know if this product respects the criteria or not. Once the reasoning process is started, these SWRL rules are applied upon the Product Profile Ontology. As long as the profile of the detergent product doesn't comply with the criteria rules, this product should be classified as an instance of "RejectedDetergent". In other words, this "RejectedDetergent" class is treated as the "objective" of the reasoning task. After the reasoning, if a product instance is classified under the type of "RejectedDetergent", we assert that this product doesn't comply with EU Eco-label criteria standard and the eco-label should not be approved.

As we interpret these rules, we should note that the concepts (classes) and relation (properties) appearing in the rules are already defined in the Criteria Ontology. They also appear in the Product Profile Ontology and the Template Ontology. Actually, the class and relation hierarchy of Product Profile Ontology and Criteria Ontology must be consistent to allow the rules in Criteria Ontology to be applied. CandidateLaundryDetergent is a class that generalizes all the detergent product before the decision making process. hasDetergentProductType is an object property specifying the product type, its domain is CandidateLaundryDetergent, range is class DetergentProductType. Under the type of DetergentProductType, 5 different product type instances (powder, liquid, gel, tablet, capsule) are defined both in Product Profile Ontology and Criteria Ontology to represent different product format. has WeightUtilityRatio is an object property specifying a product's weight/utility ratio parameter, its domain is CandidateLaundryDetergent, its range is WeightUtilityRatio. has Value is a general data property specifying quantitative parameter of certain classes. In SWRL rules, we put a question mark before a term to indicate that is a variable.

All the reasoning result and new inference of the Product Profile Ontology will be stored in the knowledge base as reference cases for further reuse or review. Thus, the knowledge base for the decision support platform will eventually be composed mainly by two parts: an ontology repository that stores all kinds of EU Eco-labeling products' ontologies in modularized way; and a historical cases repository that reserves their reasoning results and arguments.

The rest part of this section, a simple product profile example will be given to show how explanation and argumen- 
Table 2: Detailed parameters of product profile example: low-duty laundry detergent example No.0

\begin{tabular}{c|c|c}
\hline property parameter & value & criteria value \\
\hline product type & liquid & \\
sales country & France & $17.0 \mathrm{ml} / \mathrm{kg} \mathrm{wash}$ \\
recommended dosage (reference dosage) & $15.0 \mathrm{ml} / \mathrm{kg} \mathrm{wash}$ & $1.5 \mathrm{~g} / \mathrm{kg} \mathrm{wash}$ \\
weight utility ratio (WUR) & $1.4 \mathrm{~g} / \mathrm{kg} \mathrm{wash}$ & $20000.01 / \mathrm{kg} \mathrm{wash}$ \\
critical dilution volume (CDV) & $23000.0 \mathrm{l} / \mathrm{kg}$ wash & $0.30 \mathrm{~g} / \mathrm{kg} \mathrm{wash}$ \\
aerobically non-biodegradability (aNBO) & $0.25 \mathrm{~g} / \mathrm{kg}$ wash & $0.30 \mathrm{~g} / \mathrm{kg}$ wash \\
anaerobically non-biodegradability (anNBO) & $0.28 \mathrm{~g} / \mathrm{kg}$ wash \\
\hline
\end{tabular}

tation are generated at the end of reasoning. In our prototype system, the reasoning is implemented by OWL API 11 and the reasoner is Hermit ${ }^{12}$. In this example, we assume that it is a low-duty laundry detergent which is produced by Procter \& Gamble and marketed in France. Actually, one product could be marketed across several European countries at the same time. Table 2 shows the product's parameters in details.

According to the criteria, the only two known ingredients have not any hazard code, neither are they in the list of excluded or limited substances, which means they are good to be added into laundry detergent products. However, this product contains a forbidden substance EDTA (Éthylène Diamine Tétra-Acétique or Ethylenediaminetetraacetic acid), the CDV (Critical Dilution Volume) value also exceeds the criteria value, so it should be considered as a "RejectedDetergent". Figure 13 is the result snapshot from our decision support platform prototype program. In the text result showing in the console, multiple explanations are presented. The text in blue records all the 6 explanations the reasoner generates. The Hermit reasoner's explanation is originally written in Manchester Syntax ${ }^{13}$, which may be not easy to be understood by common user. We've tried to replace some of these Manchester Syntax into plain English. For example, we replace term type in Manchester Syntax with a short phrase is a type of in order to increase the explanation's readability. As we can see in Figure 13, the first explanation indicate that this product's parameters violate the rule:

hasCriticalDilutionVolume(?detergent, ?cdv), double[> "20000.0" ^double](?cdv_value), hasValue(?cdv,?cdv_value), LowDutyDetergent(?detergent $) \rightarrow$ RejectedDetergent(?detergent)

This rule specifies the Critical Dilution Volume of a low duty laundry detergent product. We can interpret this rule like this, if variable detergent is a LowDutyDetergent, detergent has Critical Dilution Volume parameter cdv, variable $c d v$ 's value is $c d v_{-}$value which is more than 20000.0, then variable detergent can be classified to be a RejectedDetergent. Apparently, as we can see from Table 2, our example product profile has critical dilution volume (CDV) of 23000.0 $1 / \mathrm{kg}$ wash, which is superior than the criteria value $20000.0 \mathrm{l} / \mathrm{kg}$ wash. It explains why this product is rejected.

Except for the first explanation in Figure 13, the other explanations concern the rule:

hasIngredient(?detergent, ?ingredient), EDT A(?ingredient), CandidateLaundryDetergent(?detergent) $\rightarrow$ RejectedDetergent(?detergent)

This rule specifies one of the forbidden ingredients that should not be added into the eco-labeled detergent product.

\footnotetext{
${ }^{11}$ http://owlapi.sourceforge.net/

12 http://www.hermit-reasoner.com/

${ }^{13}$ https://www.w3.org/TR/owl2-manchester-syntax/
} 
In human language, this rule prescribes that: if detergent has ingredient as variable ingredient, and ingredient is a kind of EDTA, then this detergent is rejected. The second ingredient of the example product is EDTA, which is forbidden as ingredient for EU Eco-labeled laundry detergent products. Therefore, this explanation indicates another reason why it is rejected.

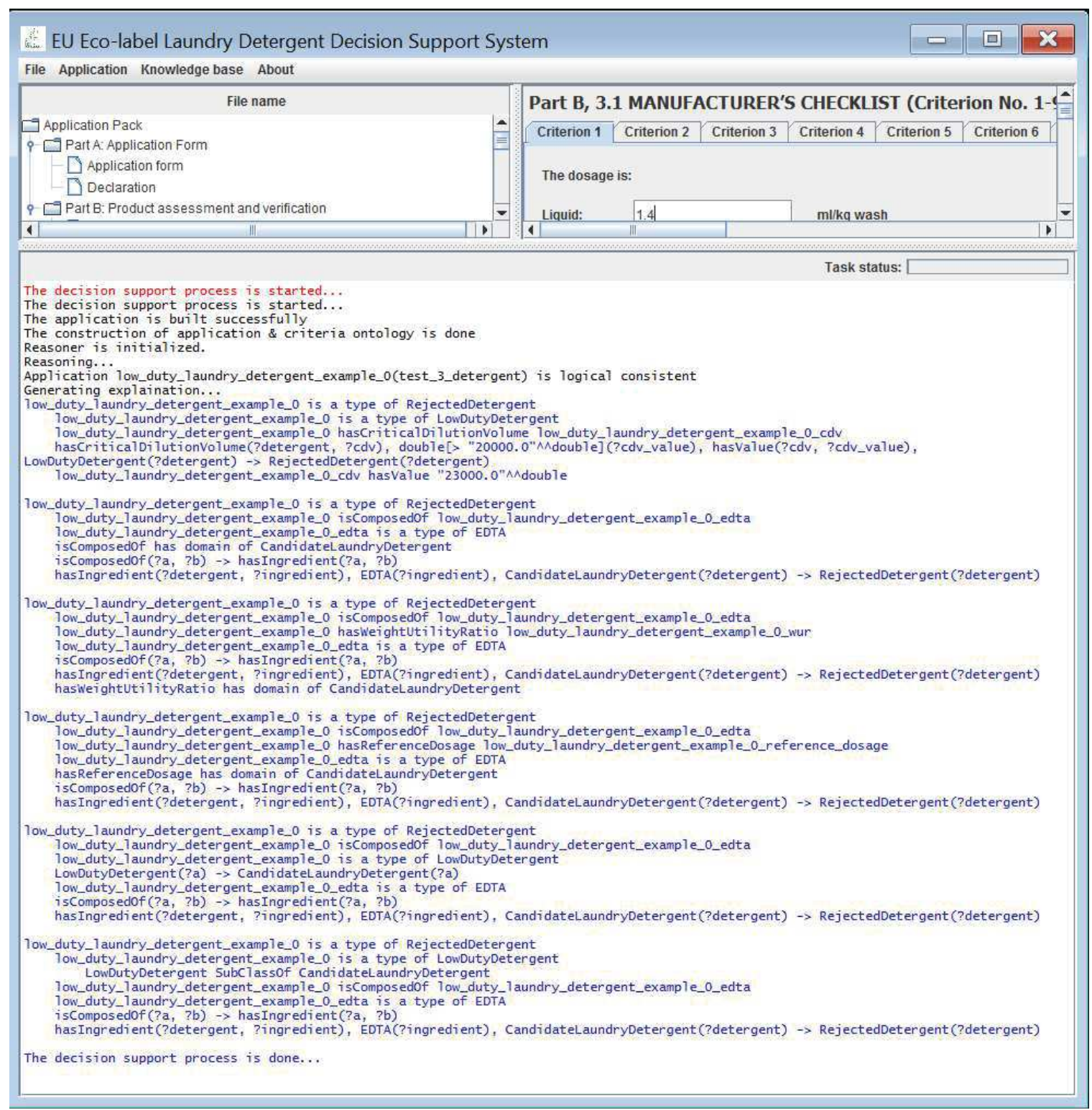

Figure 13: Reasoning result of the low-duty laundry detergent example.

As previously shown in the description of our case study and the example product profile, in case that a candidate product is rejected and argumentation is generated by our decision support tool, the decision makers can better understand why the product is rejected and which constraint or constraints of the criteria it exactly violates via reading of the explanation or argumentation of the reasoning result. 


\section{Discussion, limitation and future work}

This research is built upon the hypothesis that routine administrative communication and affairs that have few concerns to the decision making are trimmed off. Inspection and validation tasks which require much human expertise on site are also omitted. For example, the tourist accommodation or certain product's production workshop usually requires inspection on site. A textual description or even pictures are far from enough to tell if they are criteria compliant or not. Although people are pursuing for as much automatic technologies and tools as possible to accelerate the evaluation, during the general evaluation process in a broad sense, there is still some work in which human participation is essential.

The laundry detergent criteria document we use as knowledge base reference is published in Commission decision of 28 April 2011 on establishing the ecological criteria for the award of the EU Eco-label for laundry detergent 2011/264/EU. ${ }^{14}$ Most practical knowledge about laundry detergent is elaborated in the annex and appendix part. In these criterion, not all of them are suitable to be translated into ontologies. That means our system can't take over human's role completely in the decision making. Some assessment work must be conducted by man though. Take the assessment of the washing performance for example, the applicant shall provide a test report, which must be carried out by certificated laboratory, indicating that the product fulfills the minimum requirements of the test. Our system can evaluate the washing performance test result, which comes from the laboratory. Besides of some irreplaceable intervention of human labor, some complex evaluation task are still subject to human's intelligence and experience. Because a well-informed and experienced human expert is usually more competent for aesthetics and usability assessment. For example, in criterion No.8 Consumer Information, the specification of this criterion has no quantitative parameters requirement. Whether the information showing on the package is appropriate or not is mostly subject to the judgment of the human experts.

Also, from a more technical point of view, the expressiveness limit of OWL language and SWRL rules also set barrier to the full coverage of ontology knowledge base construction regarding to the criteria document. If we translate criterion document into OWL ontology forcefully and thoroughly, we may encounter very strange and complex ontology structure and semantics and that may cause extra complexity for the knowledge engineer and the reasoner in particular. For the sake of a better performance of the decision support process and our system, we propose to take a trade-off strategy that part of the criteria will be implemented by traditional program logic. This is a major limitation of our research.

For the time being, the development of the platform and the refinement of the ontologies are undergoing, e.g. the publication of the developed laundry detergent ontology modules and the SPARQL query portal introduced in section 4.2. In the next phase of our work, we will focus on more practical research and development. First, we plan to improve the argumentation generation. A more user-friendly explanation and argumentation report should be generated and Manchester Syntax should be ultimately translated into human readable language. Then, more realistic product cases should be collected and analyzed. After statistic analysis and learning, we expect our decision support platform to give improvement advice to those products that fail the eco-labeling assessment. For example, in a simulating evaluation concerning to a new laundry detergent product, if some of its ingredients are not allowed for eco-labeling, our system should give a list of feasible substitute ingredients.

In section 4.1, a modularized ontology knowledge base is presented briefly. This modular design allows a easier reusability of existent ontology modules in other product or service group. As is found in the EU Eco-label product catalog, some product groups have similarities. Take "laundry detergent" product group that has been described and

\footnotetext{
${ }^{14}$ http://eur-lex.europa.eu/legal-content/EN/TXT/?uri=CELEX:32011D0264
} 
implemented in this paper and its neighbor groups "all-purpose cleaners \& sanitary cleaners" and "rinse-off cosmetic products" for example, they share a same ingredients reference and hazard substance description. The existent ontology modules of laundry detergent can be easily reused in the construction of the other two new-coming product groups. In other words, since the rule-based reasoning mechanism of the decision support process is already established and the reasoner is independent, the functionality of our system can be extended to other product groups by adding additional criteria ontology as long as the ontology knowledge base core is still compatible with the decision support system. In our future work plan, we will develop a methodology to make our decision support system exploited by other product groups. Moreover, a more comprehensive and inter-connected ontology knowledge base based on E-Connection covering different product groups should be built.

\section{Conclusion}

In this paper, we have proposed a decision support platform trying to improve and accelerate the evaluation process for eco-labeling to help stakeholders to make wiser decisions, as well as to share knowledge and experience. From the point of view of eco-label administration domain experts, this platform is a decision support tool for the evaluation process. On the other hand, this platform can also function as a simulation tool for the producer \& service provider who wish their products or services to be eco-labeled. We have chosen EU Ecol-label as our application domain and notably the laundry detergent product group. Based on the criteria of this product group we build the knowledge base. A prototype decision support system based on modularized OWL ontology knowledge base is developed by OWL API and Jena framework. The mechanism of the decision support or simulation process is SWRL rule based reasoning and explanation. A low-duty laundry detergent product study case is presented to show how this prototype decision support system gets the product profile as input, construct relevant ontology, apply reasoning upon the ontology and finally generate explanation and argumentation in accordance to the EU Eco-label criteria. The prototype system needs further development and improvement. In our future work, we will focus more on the generation of more powerful and user friendly argumentation.

\section{References}

The EU Eco-label scheme - What is it? http://www.ecolabels.fr/en/the-eu-eco-label-scheme-what-is-it.

Facts and Figures - Ecolabel - European Commission. http://ec.europa.eu/environment/ecolabel/ facts-and-figures.html.

Global Ecolabelling Network: What is ecolabelling? http://www.globalecolabelling.net/what_is_ ecolabelling/index.htm.

Andersson, J., Johansson, B., Berglund, J., and Skoogh, A. (2012). Framework for ecolabeling using discrete event simulation. In Proceedings of the 2012 Symposium on Emerging Applications of MES in Industry and Academia Symposium, page 9 . Society for Computer Simulation International.

Baader, F. and Sattler, U. (2001). An overview of tableau algorithms for description logics. Studia Logica, 69(1):5-40.

Bao, J., Voutsadakis, G., Slutzki, G., and Honavar, V. (2009). Package-based description logics. In Modular Ontologies, pages 349-371. Springer.

Berners-Lee, T., Handler, J., and Lassila, O. (2006). The semantic web. Database and Network journal, 36(3):7. 
Blomqvist, E. (2014). The use of semantic web technologies for decision support-a survey. Semantic Web, 5(3):177-201.

Curran, M. A., Manuilova, A., Hallberg, K., Sanne, K., Widheden, J., and Bogeskär, M. (2005). Dantes: a demonstration project for sustainable development. Management of Environmental Quality: An International Journal, $16(2): 143-152$.

Da, X., Karray, H., and Archimède, B. (2016). Towards an interoperable decision support platform for eco-labeling process. In Enterprise Interoperability VII, pages 239-248. Springer.

Dreyer, L. C., Niemann, A. L., and Hauschild, M. Z. (2003). Comparison of three different lcia methods: Edip97, cml2001 and eco-indicator 99. The international journal of life cycle assessment, 8(4):191-200.

Eom, S. B. (1999). Decision support systems research: current state and trends. Industrial Management $\&$ Data Systems, 99(5):213-221.

Golden, J. S., Vermeer, D., Clemen, B., Michalko, A., Nguyen, D., Noyes, C., et al. (2010). An overview of ecolabels and sustainability certifications in the global marketplace. Corporate sustainability initiative. Durham: Nicholas Institute for Environmental Policy Solutions, Duke University.

Grau, B. C., Parsia, B., and Sirin, E. (2006). Combining owl ontologies using e-connections. Web Semantics: Science, Services and Agents on the World Wide Web, 4(1):40-59.

Grau, B. C., Parsia, B., and Sirin, E. (2009). Ontology integration using $\varepsilon$-connections. In Modular ontologies, pages 293-320. Springer.

Guarino, N., Oberle, D., and Staab, S. (2009). What is an ontology? In Handbook on ontologies, pages 1-17. Springer.

Houe, R. and Grabot, B. (2007). Knowledge modeling for eco-design. Concurrent Engineering, 15(1):7-20.

Houe, R. and Grabot, B. (2009). Assessing the compliance of a product with an eco-label: From standards to constraints. International Journal of Production Economics, 121(1):21-38.

Jolliet, O., Margni, M., Charles, R., Humbert, S., Payet, J., Rebitzer, G., and Rosenbaum, R. (2003). Impact 2002+: a new life cycle impact assessment methodology. The International Journal of Life Cycle Assessment, 8(6):324-330.

Lefébure, A. and Rosales Muñoz, R. (2011). Communicating to consumers in Sweden with eco-labels: Is the message getting through?

Leong, S., Lee, Y. T., and Riddick, F. (2006). A core manufacturing simulation data information model for manufacturing applications. In Simulation Interoperability Workshop, Simulation Interoperability and Standards Organization, pages $1-7$.

McGuinness, D. L., Van Harmelen, F., et al. (2004). Owl web ontology language overview. W3C recommendation, 10(10):2004.

Minhas, S. U. H. and Berger, U. (2014). Ontology based environmental knowledge management - a system to support decisions in manufacturing planning. In Proceedings of the International Conference on Knowledge Engineering and Ontology Development (IC3K 2014), pages 397-404.

Molander, S., Lidholm, P., Schowanek, D., Recasens, M., i Palmer, P. F., Christensen, F. M., Guinee, J. B., Hauschild, M., Jolliet, O., Pennington, D. W., et al. (2004). Omniitox-operational life-cycle impact assessment models and information tools for practitioners. The International Journal of Life Cycle Assessment, 9(5):282-288. 
Parent, C. and Spaccapietra, S. (2009). An overview of modularity. In Modular Ontologies, pages 5-23. Springer.

Pathak, J., Johnson, T. M., and Chute, C. G. (2009). Survey of modular ontology techniques and their applications in the biomedical domain. Integrated computer-aided engineering, 16(3):225-242.

Serafini, L. and Tamilin, A. (2005). Drago: Distributed reasoning architecture for the semantic web. In The Semantic Web: Research and Applications, pages 361-376. Springer.

Serafini, L. and Tamilin, A. (2009). Composing modular ontologies with distributed description logics. In Modular Ontologies, pages 321-347. Springer.

Shearer, R., Motik, B., and Horrocks, I. (2008). Hermit: A highly-efficient owl reasoner. In OWLED, volume 432, page 91 .

Sirin, E., Parsia, B., Grau, B. C., Kalyanpur, A., and Katz, Y. (2007). Pellet: A practical owl-dl reasoner. Web Semantics: science, services and agents on the World Wide Web, 5(2):51-53.

Studer, R., Benjamins, V. R., and Fensel, D. (1998). Knowledge engineering: principles and methods. Data $\mathscr{6}$ knowledge engineering, 25(1):161-197.

Tsarkov, D. and Horrocks, I. (2006). Fact ++ description logic reasoner: System description. In International Joint Conference on Automated Reasoning, pages 292-297. Springer.

Vilhelmiina Ihamäki, Eva van Ooij, S. v. d. P. (2014). Green public procurement in the european union and the use of eco-labels.

Whyte, R. G. (1986). What is a decision support system? Industrial Management E Data Systems, 86(7/8):28-30. 